\title{
eJRIEPS
}

Ejournal de la recherche sur l'intervention en éducation physique et sport

$23 \mid 2011$

Varia

\section{Analyse technologique du rôle de libéro en volley- ball : une influence paradoxale sur le jeu}

\section{Serge Eloi et J-F Robin}

\section{OpenEdition}

Journals

Édition électronique

URL : http://journals.openedition.org/ejrieps/4545

DOI : 10.4000/ejrieps.4545

ISSN : 2105-0821

Éditeur

ELLIADD

\section{Référence électronique}

Serge Eloi et J-F Robin, « Analyse technologique du rôle de libéro en volley-ball : une influence paradoxale sur le jeu », eJRIEPS [En ligne], 23 | 2011, mis en ligne le 01 avril 2011, consulté le 21 février 2020. URL : http://journals.openedition.org/ejrieps/4545 ; DOI : 10.4000/ejrieps.4545

\section{(c) (1)}

La revue eJRIEPS est mise à disposition selon les termes de la Creative Commons Attribution 4.0 International License. 
Analyse technologique du rôle de libéro en volley-ball : une influence paradoxale sur le jeu

\section{Serge Eloi}

Université Paris-Est Créteil Val-de-Marne, Laboratoire REV/CIRCEF, 61 avenue du Général de Gaulle 94010, Créteil Cedex, France

\section{Résumé}

Dans cette étude, notre objectif est de montrer les éléments antithétiques qui apparaissent dans l'évolution du règlement de volley-ball. Plus particulièrement, nous voulons montrer en quoi l'apparition du libéro en 1999 constitue un paradoxe dans la longue liste des changements réglementaires qui ponctuent l'histoire du volley-ball. Nous tenterons de placer le lecteur dans le dilemme vécu par ceux qui doivent prendre la décision de modifier (ou non) les lois du jeu. Pour ce faire, nous avons choisi d'adopter une démarche technologique. La spécificité d'une telle démarche se caractérise par la nécessité de se référer aux différents registres de technicité (Martinand, 1994) et d'identifier les artefacts mobilisés (Rabardel, 1995 ; Vérillon, 2005). Nous serons donc amenés à faire le lien entre les outils et leur mode d'utilisation. L'objectif poursuivi est de livrer les logiques clefs qui permettent l'accès à une conception dynamique des lois du jeu. La démarche technologique ambitionne d'aller au-delà de l'explicitation de la logique d'évolution du système. Elle vise notamment à "irriguer" à nouveau le terrain. Nous ferons pour conclure une proposition argumentée de modification du règlement en direction des législateurs.

\section{Introduction}

Notre travail se situe dans le cadre d'une recherche technologique (Ulrich \& Éloi ; Éloi \& Ulrich, dans ce numéro) et fait suite à la réflexion impulsée par Vigarello (1988) puis par Durey et Bouthier (1994). Les problématiques sont donc issues du terrain pour aboutir à des propositions qui ont pour ambition d'aller à nouveau investir le terrain. Soit sous forme d'analyses et d'explicitations : les résultats de ces investigations sont dans ce premier cas, réintroduis dans le cadre des formations. Soit sous forme de propositions de remédiations: les résultats de la recherche technologique aboutissent alors à la construction d'outils (schémas, matériels spécifiques, logiciels informatiques, etc.) ou de dispositifs (organisation d'une simulation, formalisation d'une situation, etc.). Ces procédés 


\section{eJRIEPS 23 avril 2011}

sont dans ce second cas soumis aux intervenants pour tenter de résoudre les problèmes évoqués. C'est donc dans une relation dialectique effective que doivent être conçues ces nouvelles créations. Nous souhaitons participer à « une démarche importante, précieuse, nouvelle elle aussi, pour la compréhension de la technique et de ces usages » qui pourtant « demeure curieusement négligée, ou ignorée, dans le champ des APS : celle de la «technologie culturelle » (Vigarello, 1991, p. 146. Nous nous proposons donc d'utiliser la démarche technologique pour mettre à jour les contradictions qui sont apparues dans le processus de développement de la règle au volley-ball. En effet, avec l'apparition du libéro se pose le problème de la continuité de la logique d'évolution du règlement. Alors que cette logique reposait jusqu'alors sur des tentatives de rééquilibration du rapport attaque/défense (introductions de règles en faveur de la défense historiquement déficitaire), il semble que l'apparition du rôle de libéro entre en contradiction avec une telle conception. Notre propos suivra donc deux axes :

- d'une part, il s'agira de spécifier en quoi la démarche technologique permet l'analyse d'un tel cas de figure. La méthode étant novatrice, nous décrirons les concepts utilisés à cet effet. Nous reviendrons ensuite sur la façon dont ils ont été mobilisés au travers de cette étude.

- D'autre part, nous nous appuierons sur les résultats de diverses recherches et de données issues des règlements successifs pour étayer la thèse de la rupture. Nous montrerons comment cette rupture de la logique historique de développement de la règle au volley-ball conduit à une impasse dont on pourrait néanmoins sortir. Cette issue sera proposée sous la forme d'une modification d'un article du règlement.

\section{La démarche technologique : un guide pour l'analyse du rôle de libéro}

Adopter une démarche technologique nous engage à répondre à quatre questions :

- Quelle est la visée de la recherche technologique?

- Quels sont les liens entre registre principal, registres associés et artefacts mobilisés ?

- Quelles sont les connaissances mobilisées?

- À quelles propositions aboutit la recherche?

1. 1. Une visée

Dans cette étude, notre objectif est de montrer les éléments antithétiques qui apparaissent dans l'évolution du règlement de volley-ball. II s'agit de mettre à jour les diverses influences qui structurent le système complexe que constitue la règle (Le Moigne, 1999). Plus précisément, notre ambition est de dévoiler les contradictions inhérentes au développement d'une activité sportive socialement partagée. Nous tenterons donc de 


\section{eJRIEPS 23 avril 2011}

placer le lecteur dans le dilemme vécu par ceux qui doivent prendre la décision de modifier (ou non) les lois du jeu.

1. 2. Une méthode : la sollicitation complémentaire des registres et des artefacts La démarche technologique nécessite de se référer à des registres de technicité (Martinand, 1994) et d'identifier les artefacts mobilisés (Rabardel, 1995 ; Vérillon, 2005). En effet, il apparait que c'est par la mobilisation judicieuse d'artefacts qu'il devient possible de faire avancer les connaissances dans un registre identifié. Une telle entreprise requiert d'une part, d'indiquer quel est le registre principal et quels sont les registres associés. Nous nous inscrivons donc dans une perspective dynamique de cette catégorisation. Loin de considérer que les registres sont cloisonnés, il nous semble au contraire opportun d'envisager les influences réciproques des uns sur les autres. Les découvertes faites dans l'un des registres pouvant être un élément d'explication pour les problématiques relevées dans les autres. D'autre part, il s'agira d'identifier les artefacts mobilisés au cours de la recherche. Dans la mesure du possible, il serait souhaitable de montrer la relation entre les résultats escomptés et la mobilisation de tels outils. Nous avons précédemment relevé (Ulrich \& Éloi, dans ce numéro) que quatre types de registres pouvaient permettre de caractériser les recherches technologiques en APSA : les registres de maîtrise, de lecture, de participation et de transformation. Consécutivement (Éloi et Ulrich, dans ce numéro), nous avons proposé de faire référence à quatre classes d'artéfacts pour couvrir le champ de l'intervention en APSA : les artéfacts cognitifs, corporels, matériels et réglementaires. Nous proposons de caractériser notre travail en référence à ces catégories.

\section{2. 1. Registre principal et registre associés}

Le registre principal mobilisé dans notre analyse est le registre de participation. En effet, il relève de l'analyse de l'activité déployée en dehors de la pratique de la discipline sportive en elle-même. Au-delà des membres de la commission des lois du jeu, les acteurs qui contribuent à l'évolution de la règle sont nombreux. Les joueurs bien sûr qui poussent toujours à la limite les lois existantes par le développement permanent de leurs habiletés tactico-techniques et l'augmentation constante de leurs capacités physiques. Les entraineurs qui doivent s'emparer de la règle pour l'exploiter au mieux. Mais aussi les dirigeants des fédérations qui tentent d'orienter les évolutions en fonction des spécificités du jeu de leur pays. II serait donc naïf de penser que les modifications du règlement se font en dehors de tout lobbying. Cependant, plutôt que de formuler des hypothèses sur les raisons réelles qui aboutissent au vote des nouvelles dispositions, nous nous attacherons à montrer les conséquences que les règles successives ont eues sur le jeu. C'est dans ce cadre que nous solliciterons particulièrement deux registres associés. D'une part, le 


\section{eJRIEPS 23 avril 2011}

registre de maîtrise car il prend notamment en compte les états de contrôle des techniques. Il est fait référence ici aux exigences de contrôle du ballon dans des situations caractéristiques du jeu. Au sein de ce registre est développé l'argumentaire qui permet d'orienter le choix du profil des joueurs recrutés. D'autre part, le registre de transformation qui regroupe les données relatives aux conditions et procédures d'évolution des savoirs tactico-techniques. II a trait aux moyens que joueurs et entraineurs convoquent pour s'approprier ces nouvelles solutions qui permettront l'adaptation aux nouvelles règles.

\section{2. 2. Les artefacts mobilisés}

Lors de notre démonstration, nous serons amenés à employer deux types artefacts.

- Des artefacts cognitifs : éléments artificiels et conceptuels conçus pour traiter l'information dans le but de satisfaire une fonction représentationnelle, ces artefacts permettent de clarifier les termes de la problématique. Nous les utiliserons notamment pour tenter de modifier des représentations largement répandues bien que non fondées.

- Des artefacts matériels : Là où le discours écrit s'avère incertain, le schéma permet de saisir rapidement les données du problème. Les schémas employés ici sont donc des outils techniques chargés de conceptualisation. De fait, l'élaboration de l'outil a nécessité de rationaliser les différentes formes de pensées relatives à l'évolution du règlement. Par conséquent, il dépasse un simple plan de situation.

1. 3. Des connaissances mobilisées : référence à des problématiques, des concepts ou des normes.

II sera fait référence ici à des concepts issus notamment des travaux de René Deleplace. En effet, cet auteur a su développer une modélisation pour les sports collectifs (Deleplace, 1983) qui nous permet de structurer la réflexion que nous menons à propos du volley-ball. Ainsi, nous serons amenés à mobiliser un schéma de modélisation qu'il nous faudra cependant développer pour «coller» aux particularités de notre discipline. Par ailleurs, d'autres représentations graphiques ont été construites dans le but de permettre de saisir la spécificité du rapport d'opposition propre au volley-ball.

\section{4. Des propositions pour la diffusion}

Au delà de la compréhension du système complexe qui abouti à l'évolution du règlement, notre démarche vise à « irriguer » à nouveau le terrain. D'une part, nous aurons atteint notre objectif si nous réussissons à amener le lecteur à avoir une conception dynamique des lois du jeu. D'autre part, pour conclure cette recherche, nous nous risquerons à faire quelques suggestions en direction des législateurs. Nous argumenterons alors pour justifier cette proposition de modification du règlement. 


\section{Modélisation du règlement de volley-ball}

$\mathrm{Si}$, d'une façon générale, la règle prend acte des innovations techniques des pratiquants en les entérinant, il est des contextes où les transformations réglementaires sont le fruit de délibérations qui sont guidées par des logiques plus éloignées du terrain. Pour Vigarello, «le règlement est souvent plus qu'un simple «résultat». II est un compromis, une synthèse. Le règlement est un point de rencontre entres des histoires différentes. II en est aussi, dans une certaine mesure, l'issue " (Vigarello, 1988, p. 188). Le volley-ball est un bel exemple de ces compromis. Et pour cause. II est assurément le sport collectif dont le règlement a subi le plus d'évolutions lors des deux dernières décades. Le summum fut probablement atteint pendant la saison 1998-1999 au cours de laquelle (entre autres) le système de décompte des points fut radicalement changé. En effet, les championnats de France professionnels ont alors suivi un scénario insolite. Au cours des matchs allers, c'est avec l'ancien système de décompte des points que les rencontres se sont déroulées (il fallait être au service et remporter l'échange pour marquer un point). Puis, lors des matchs retours c'est le nouveau système de décompte des points qui fut adopté (quelles que soit l'équipe au service, l'équipe qui remporte l'échange marque un point). Nous ne reviendrons pas ici sur les réactions provoquées dans le milieu à cette occasion. Le nouveau système de décompte des points (Rally Point System ou marque continue) étant accusé d'altérer le championnat. La presse se fit alors l'écho de ces réactions en les amplifiant parfois. II devait y avoir nivellement des équipes, les meilleures se trouvant lésées et les moins bonnes favorisées. En fait, nous avons mené à l'époque une étude qui a révélé que la hiérarchie n’a en rien été bousculée (Éloi, 1999). C'est dans ce contexte particulier que le rôle de libero est apparu. Plus précisément, c'est lors des matchs retours de cette saison (1998-1999) que ce profil de poste s'est mis en place. Or, à cette époque, notre étude qui avait pour but de déterminer si un tel changement pouvait avoir une influence sur la hiérarchie des équipes (Éloi, 1999) a révélé que sur 156 possibilités d'inscrire le libéro sur la feuille de match, 92 seulement furent utilisées (comptage fait sur l'ensemble des feuilles des matchs retours de la Pro A masculine, saison 1998-1999). Aujourd'hui, sauf circonstances exceptionnelles, toutes les équipes utilisent un libéro. Cette évolution se trouve confortée par le règlement de la saison dernière (FIVB, 20092012). En effet, alors qu'un seul joueur pouvait remplir ce rôle au cours d'une rencontre, privant l'équipe de ce poste en cas de blessure dudit joueur, le règlement prévoit maintenant qu'un libéro remplaçant peut être inscrit sur la feuille de match. Notre thèse consiste à montrer que l'apparition de ce rôle très particulier dans une équipe de volley- 


\section{eJRIEPS 23 avril 2011}

ball et sa confirmation récente vont à l'encontre de la logique de développement de la règle identifiée jusqu'alors. II nous faudra auparavant en passer par d'indispensables prolégomènes afin d'exposer les principaux éléments de la modélisation du règlement de volley-ball.

En nous appuyant sur les travaux de René Deleplace (1979), nous avons proposés de modéliser les sports collectifs en distinguant deux secteurs : d'une part, le règlement et d'autre part, les conduites tactiques (Éloi et Ulrich, 2001). Nous nous limiterons à la modélisation du règlement dans le cadre de cet article. Traditionnellement, les lois du jeu sont présentées sous la forme d'articles successifs organisés en grandes thématiques permettant d'utiliser le recueil comme un dictionnaire. Deleplace a montré que les règles pouvaient s'articuler en une toute autre logique (Deleplace, 1979, 1983). Ses travaux conduisent à appréhender la règle comme un ensemble constitué d'un noyau central et de règles complémentaires. D'un côté, des règles immuables qui constituent «le code génétique » de la pratique sportive. De l'autre, des règles additionnelles qui permettent, tout au long du développement historique de la discipline, son adaptation aux évolutions stratégico-tactiques, tactico-techniques ou physiques des joueurs et du jeu.

2. 1. Double logique de composition du règlement

«La règle a pour fonction de faire se reproduire le jeu » (Deleplace, 1983, p.100). Car si le jeu a toujours précédé ontogénétiquement la règle (David, 1993), le jeu ne peut vivre que par la règle. Coulon défend l'hypothèse d'un double caractère des règles. Celui « d'être à la fois des règles techniques régissant le jeu, et des règles sociales qui lui permettent de se dérouler concrètement, ... »(Coulon, 1991, p. 185). Et il nous semble bien que la reproduction du jeu ne peut se faire que dans le compromis de cette double nécessité. En effet, la reproduction mais aussi le développement du jeu doivent être possible en dépit du temps et des lieux dans lesquels il se déroule.

- Le temps : la règle doit conserver sa substantifique essence tout en prenant en compte les évolutions liées aux divers développements (physiques, stratégiques, tactiques, techniques).

- Les lieux : il doit être possible d'organiser des rencontres entre protagonistes originaires de tous les pays. Ainsi, la Fédération Internationale de Volley-ball (FIVB) unifiera les règlements issus des différentes zones en 1947.

Cette double exigence «spatio-temporelle» conduit alors à une double logique de composition du règlement. D'une part, des règles qui ont pour vocation la préservation des caractéristiques profondes du jeu en tant que discipline originale au sein d'une famille plus large (les sports collectifs). C'est le noyau central du règlement. D'autre part, des règles 


\section{eJRIEPS 23 avril 2011}

permettant l'adaptation intelligente de ce noyau central en fonction des inventions et innovations mises en œuvre par les acteurs du mouvement sportif (joueurs, entraîneurs ou dirigeants). Ce sont les règles complémentaires.

\section{2. Le noyau central du règlement (NCR)}

II s'agit du noyau historiquement stable du volley-ball. II est le garant de sa logique spécifique. De ce fait, les règles qui le composent ne peuvent être changées sans porter fatalement atteinte à l'essence même du jeu. II comporte les indications relatives au but poursuivi par les protagonistes ainsi que leurs droits essentiels (droits liés à l'atteinte du but). Au volley-ball il peut se définir ainsi :

Le but du jeu :

- il s'agit de faire tomber la balle dans le camp adverse (1895).

Les droits essentiels des joueurs :

- la balle doit être frappée donc les espaces de jeu sont séparés (1895). Le filet est garant de la séparation des espaces de jeu (voir Éloi \& Ulrich (2001) pour une analyse plus détaillée).

Afin qu'il n'y ait pas monopolisation du ballon :

- chaque joueur ne peut jouer deux fois consécutivement la balle (1917 aux États-Unis et 1920 en France).

- chaque équipe dispose de 3 touches de balle pour atteindre le camp adverse (1920).

Notons que cette dernière règle marque pour nous la naissance véritable du volley-ball en tant que sport moderne. Du point de vue didactique, ce noyau central du règlement contribue à donner une représentation du jeu directement orientée vers la prise en compte de l'adversaire. II peut d'ailleurs être délivré dés la toute première initiation. A cette occasion, l'intervenant en dévoilant ces quatre règles et en expliquant la relation dialectique qui les unit permet aux débutants d'accéder immédiatement à la logique spécifique du jeu. Cette logique peut s'exprimer de la façon suivante. « La configuration matérielle des cibles détermine un affrontement original puisque les espaces de jeu sont séparés par un filet. L'impossibilité pour l'adversaire de venir chercher le ballon a comme corollaire l'impossibilité de bloquer la balle. La balle doit donc être frappée » (Éloi \& Ulrich, 2001, p.118). Ce n'est qu'ensuite, au cours de l'apparition aléatoire des faits de la pratique que des précisions réglementaires pourront être apportées par l'intervenant.

\section{3. Les règles complémentaires}

Elles sont apparues tout au long du développement du volley-ball. Et c'est seulement avec le recul historique que l'ont peut affirmer que les législateurs ont cherché à garantir un attrait ludique constant en tentant de préserver l'équilibre entre l'attaque et la défense. Car 


\section{eJRIEPS 23 avril 2011}

il y a bien un « enjeu vital » pour le jeu à mettre en place un cadrage réglementaire qui préserve à tout moment les chances de chacun. À ce propos, Bernard Jeu nous dit que « l'essentiel, ce que l'attention retient, c'est la mise en face l'un de l'autre de deux héros, de deux champions, de deux équipes, le moment précis où l'on va basculer de l'égalité des chances vers l'inégalité du résultat. C'est le tragique séparé du destin, dans le moment privilégié de sa liberté et de son incertitude » (Jeu, 1983, p. 31). Il est ici suggéré que pour que le jeu tienne toutes ses promesses, c'est à dire, pour que le caractère ludique perdure, il est nécessaire que les conditions initiales d'équilibre soient remplies. C'est alors au cours d'une rencontre particulière, à l'occasion d'une confrontation singulière que les vainqueurs se dévoileront. La seconde partie de la citation (souvent délaissée) consacre cette idée. Car si la mise en scène qui est organisée vise à la mort symbolique (la défaite) d'une des parties, elle doit le faire sans qu'un quelconque déterminisme ne puisse interférer. C'est donc en êtres libres que les protagonistes viennent se confronter, acceptant de fait l'incertitude liée à l'issue du combat qui va les opposer. II est donc crucial pour que vive le jeu que l'équilibre entre l'attaque et la défense soit le plus accompli. C'est la raison pour laquelle il est nécessaire de déterminer qui de l'attaque ou de la défense prend le pas. Et il existe un moyen très simple de le savoir au volley-ball. II suffit de compter le nombre de fois où l'équipe au service remporte l'échange. L'article 6.1.3 du règlement intitulé «Echange de jeu et échange de jeu terminé » définit un échange comme suit : «Un échange de jeu est la séquence d'actions de jeu à partir de la frappe de service par le serveur jusqu'à ce que le ballon soit hors jeu. Un échange de jeu terminé est la succession d'actions de jeu dont le résultat est le gain d'un point »(Règle FIVB, 2009-2012, p. 14). Or, à haut niveau, l'essentiel des échanges a pour caractéristique que la balle passe à peine plus de deux fois le filet. Selon une étude de Fournier, cette moyenne était de 2,39 en 2000 et de 2,31 en 2002 (Fournier, 2005). Le premier passage de filet s'effectue lorsque le serveur réussi son service. Le second lorsque l'un des attaquants de l'équipe en réception attaque. « L'introduction de la règle du libéro n'a donc pas eu pour effet d'augmenter le nombre d'échanges, mais de le réduire significativement dans un premier temps et peu significativement ensuite » (Fournier, 2005, p. 132). Cette donnée s'explique par le fait que le déséquilibre attaque/défense est tel que la première attaque est le plus souvent décisive. Ainsi, dans deux cas sur trois l'équipe qui initie l'échange le perd. Dans une étude menée lors de la saison (2009-2010), nous avons pu relever que le rapport « nombre de points gagnés/ nombre d'échanges initiés » avait été de 0,38 points en moyenne pour l'équipe de Tours qui a été championne de France (Albert, 2010). Cette donnée correspond à 1,14 point 


\section{eJRIEPS 23 avril 2011}

marqué sur 3 services réalisés. À ce stade de l'argumentation, il n'est pas inutile d'illustrer pourquoi l'équipe dont l'un des joueurs est au service est celle qui est en défense. De fait, immédiatement après la mise en jeu, trois cas de figure peuvent se présenter. Première possibilité, le serveur manque son service (balle dans le filet, balle « out », faute réglementaire). II n'y a pas eu d'intervention des adversaires et c'est à l'équipe adverse de servir. Deuxième possibilité, le serveur marque directement le point (ace ou service gagnant). Dans ce cas, l'intervention des adversaires se limite à sa plus simple expression (ace : regarder la balle tomber hors de portée ; service gagnant: le réceptionneur ne parvient pas à contrôler le service qui est injouable par un partenaire) et c'est le même serveur qui sert à nouveau. Troisième possibilité, il y a réception de l'équipe adverse qui s'organise pour attaquer. L'équipe du serveur doit alors défendre. C'est donc en toute logique que l'équipe qui sert est l'équipe en défense. Relever un tel état de fait conduit à mettre en évidence un déficit important de la défense par rapport à l'attaque en volley-ball. En effet, si l'équilibre idéal se situe dans une proportion de un sur deux (un service sur deux aboutit au gain de l'échange), il est patent de constater que pour l'équipe la plus performante de notre championnat, elle se situe juste au-dessus de un sur trois.

Et il semble bien que ce déficit soit une constante « génétique » du volley-ball car bien que les législateurs successifs aient tenté au travers de l'évolution du règlement de contrecarrer cette disproportion, le déficit perdure. Que serait-il advenu si les règles étaient restées inchangées ?

Demiselle note que « cette recherche d'adaptation systématique d'un secteur par rapport à l'autre tente d'équilibrer l'attaque et la défense. Mais, aujourd'hui encore, l'équilibre du rapport de forces n'est pas acquis; les phases offensives ont toujours le dessus » (Demiselle, 2005, p. 236). Ainsi, depuis toujours, les membres de la commission des lois du jeu ont emprunté deux voies complémentaires pour tenter de remédier à cet état de fait. D'une part, ils ont promulgué des lois du jeu visant à augmenter le pouvoir de la défense. D'autre part, ils ont prescrit des règles diminuant le pouvoir de l'attaque. 


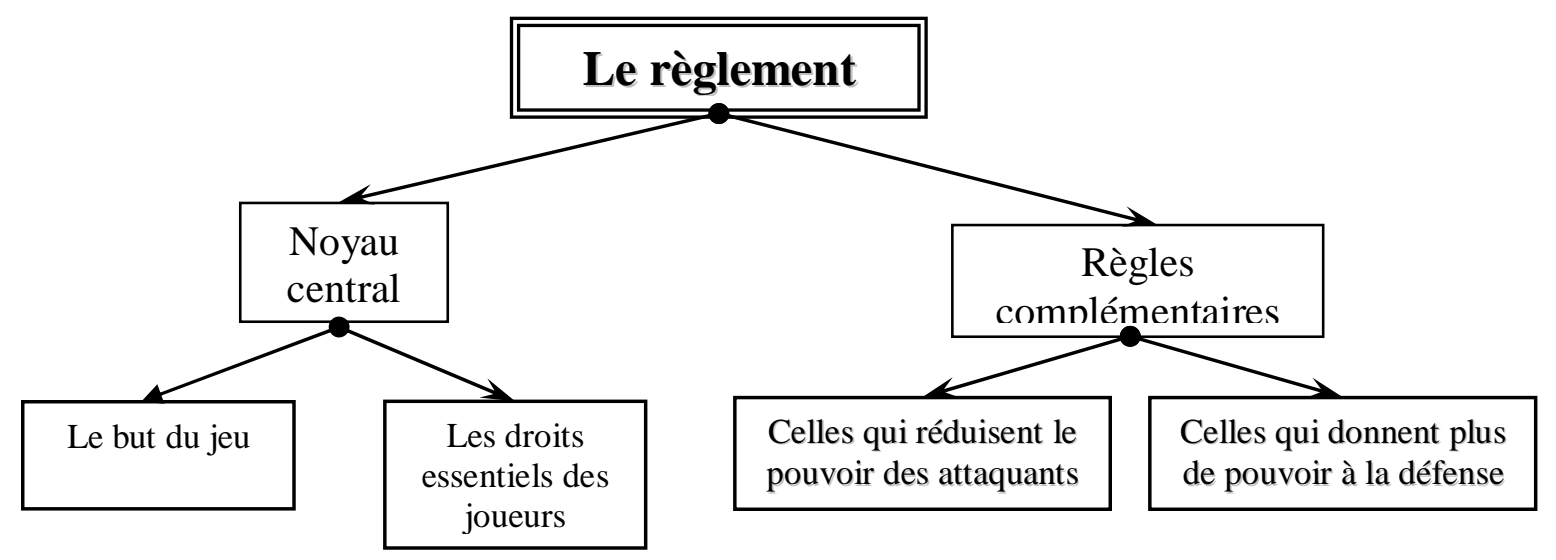

$\underline{\text { Figure } 1}$ : schéma provisoire de modélisation de la règle au volley-ball

Pour illustrer notre propos, nous nous limiterons ici à donner quelques exemples distribués dans le temps.

2. 3. 1. Les règles complémentaires qui augmentent le pouvoir des joueurs en défense.

Au volley-ball, les actions défensives sont réparties entre le contre (intervention au dessus du filet qui a pour vocation de renvoyer directement l'attaque de l'adversaire dans son camp) et la défense basse (plus éloignée du filet qui consiste dans un même temps à empêcher que la balle ne tombe et à la récupérer pour contre-attaquer).

- L'autorisation est faite aux contreurs de passer les mains au-dessus du filet (1964). En positionnant ses mains de l'autre côté du filet, le contreur les rapproche du ballon. II réduit ainsi l'angle possible d'attaque du smasheur. De plus, l'angle d'incidence des bras tend à rabattre la balle vers le sol ce qui rend plus problématique le soutien du joueur qui attaque ${ }^{1}$.

- Reprise de balle par le contreur (doublé) (1970). Considérant que le contreur n'est pas maître de sa touche de balle (il subit le smash adverse) l'autorisation est donnée au contreur de jouer le ballon immédiatement après son contre. II peut donc doubler la touche de balle.

- La touche de balle effectuée par un contreur n'est pas comptabilisée (1976). Cette règle entérine le fait que le contre est une action très particulière. En effet, toute attaque nécessite une touche de récupération, une touche de passe et une touche de renvoi (smash). En permettant que la touche de contre ne soit pas comptabilisée, il devient

\footnotetext{
${ }^{1}$ Le soutien est l'action qui consiste à éviter qu'une frappe d'attaque contrée ne retombe dans son propre camp. Les partenaires du smasheur, en se répartissant autour de lui au moment du smash, s'organisent pour pallier les conséquences d'un contre.
} 


\section{eJRIEPS 23 avril 2011}

possible d'organiser véritablement la contre-attaque. Cette règle donne un avantage supplémentaire à la défense.

- La possibilité de touches simultanées sur réception et première défense (1992). Cette règle prend en compte que les frappes de balles sont devenues de plus en plus puissantes et donc beaucoup plus difficiles à contrôler. Elle augmente la tolérance relative au contact de balle.

- Dans le même ordre d'idée, on assiste à l'augmentation des surfaces de frappe : jusqu'à la ceinture initialement puis, jusqu'aux genoux (1992) et enfin, toutes les parties du corps (1994).

\section{3. 2. Les règles qui réduisent les droits des joueurs à l'attaque.}

- L'apparition de la ligne des $3 \mathrm{~m}$ qui contraint les arrières à attaquer derrière cette démarcation diminuant ainsi le nombre d'attaquants proches du filet (1922).

- La réduction de l'espace de passage du ballon au-dessus du filet par l'adjonction de mires à l'intersection des lignes au sol et du plan du filet. Cette réduction s'est faite en deux temps. Tout d'abord (1972), la mire fut placée $25 \mathrm{~cm}$ à l'extérieur de la ligne de côté du terrain ce qui correspond au diamètre du ballon (l'écart entre les deux mires était de 9,5 m). Puis, à l'olympiade suivante (1976), il fut considéré que la totalité du ballon devait passer à l'intérieur de l'espace de jeu. Les mires furent alors alignées avec l'extérieur des lignes de côtés au sol (L'écart entre les mires est ramené à $9 \mathrm{~m}$ ).

II est patent de constater que les règles qui réduisent les droits des attaquants sont beaucoup moins nombreuses que celles qui augmentent le droit des défenseurs. On doit certainement y voir la volonté de conserver le caractère spectaculaire des frappes de balles aériennes. En suivant l'historique des règles on peut saisir la logique de la commission des lois du jeu de la FIVB qui tente de rééquilibrer le rapport attaque-défense. Cependant, les velléités des législateurs n'ont pas été suffisantes puisqu'un déséquilibre important perdure. II apparaît parallèlement que les membres de la commission des lois du jeu se sont employés, lors de ces deux dernières décennies, à rendre le spectacle «volley-ball» de plus en plus attrayant. Noble visée qui n'est cependant pas sans conséquences.

\section{Le développement médiatique du volley-ball}

Toute activité sportive à pour vocation de se développer et l'un des vecteurs essentiels de communication moderne est son image. Pour Vigarello, « Avant d'être consommation, l'image est devenue depuis peu, le support d'une promotion et d'une valorisation de l'objet. Elle lui donne même quelques fois existence et sens. Mode de circulation des 


\section{eJRIEPS 23 avril 2011}

choses, véhicule du lien social, elle prend bien souvent la place des faits eux-mêmes » (Vigarello, 1988, p. 149). Or il s'avère qu'un déficit d'image important est subi par le volleyball en France. Ainsi, lorsqu'avec l'équipe du Paris Volley nous avons remporté la ligue des clubs champions à Paris (Halle Carpentier, 2001), il ne fut pas possible de faire retransmettre la finale sur une chaine non cryptées. De la même façon, lorsqu'en 2009, l'équipe de France masculine a joué la finale des championnats d'Europe des Nations, la finale ne fut visible que sur une chaine câblée. Peut-on imaginer qu'un tel fait soit possible dans d'autres disciplines ? Cette situation qui n'est pas spécifique à la France a donc enjoint nos dirigeants à tenter de rendre notre activité plus attrayante. C'est pour cette raison que les instances internationales se sont évertuées à faire évoluer le mode de déroulement des rencontres. Philippe Blain, entraineur de l'équipe de France et Président de la commission des entraineurs de la FIVB nous rappelle « la nécessité dans un futur proche :

1. D'améliorer l'aspect spectaculaire de notre sport pour les fans, sponsors et médias

2. D'accroître le nombre d'échanges de balle au court d'un même point

3. De garder les même règles pour toute la famille du volley-ball quelque soit l'âge ou le sexe » (Blain, 2010, p. 32).

\section{1. Problématique médiatique}

Il y a une double difficulté à faire du volley-ball un produit « télévisuellement » conforme. D'une part, il faut réduire la durée globale des rencontres. D'autre part, il faut maîtriser l'éventualité d'un écart entre une rencontre courte (en 3 sets) et une rencontre longue (en 5 sets). En effet, il est souvent évoqué par les programmateurs des chaines de télévision que ces aléas empêchent toute programmation puisqu'il est impossible de prédire la durée et donc, l'heure de fin d'une rencontre. Vigarello (1988) relève qu'une pression grandissante est exercée sur les « concepteurs de jeu » qui ambitionnent pour leur sport une plus grande présence télévisuelle. Elle les oblige à des inventions réglementaires qui ne sont pas sans répercutions sur les évolutions techniques. Dans ce cadre, la maîtrise des temps de jeu est un atout majeur pour infiltrer les programmes. L'une des premières règles énoncées pour réduire la durée globale d'un match fut la règle dite des 3 ballons (1978). Un gain de temps non négligeable (on parle de $20 \%$ de temps gagné) fut constaté suite à la mise à disposition d'un ballon du côté des serveurs. Par l'intermédiaire de quatre ramasseurs de balles, un ballon se trouve en attente à chaque extrémité du terrain. Le ballon utilisé lors de l'échange précédant étant ramené du côté du dernier serveur durant l'échange en cours. Cependant, cette réduction ne suffisait pas et ne permettait pas de 


\section{eJRIEPS 23 avril 2011}

contrôler l'écart entre un match en 3 sets et un match en 5 sets. II a donc fallu prendre des mesures supplémentaires.

3. 2. Développement de la première modélisation

L'exemple donné précédemment a dû interpeller le lecteur attentif. Nous avons en effet modélisé le règlement en deux catégories: le noyau central du règlement et les règles complémentaires. Puis nous avons donné les principes de constitution de ces deux catégories.

Or, il semble bien que cette règle dont la vocation est de réduire le temps de jeu n'influence en rien le rapport attaque-défense. C'est la raison pour laquelle il nous faut modifier notre représentation de la modélisation de la règle de volley-ball. La volonté de rendre plus spectaculaire et médiatique notre discipline a conduit à promulguer un certain nombre de lois du jeu que l'on peut regrouper au sein d'une nouvelle catégorie : les règles complémentaires indépendantes du jeu. Ces nouvelles catégories illustrent concrètement les propositions faites par Vigarello lorsqu'il indique que « le règlement est au point d'intersection de dynamiques le plus souvent séparées. Sa formulation indique l'emprise de chacune d'elles, elle révèle leur poids respectif » (Vigarello, 1988, p. 190). C'est la distribution de ces différentes influences que nous nous efforçons de mettre à jour ici. Ainsi les règles complémentaires sont constituées de deux sous catégories. D'un côté, les règles complémentaires inhérentes au jeu qui ont pour objectif de rééquilibrer le rapport attaque-défense. De l'autre, les règles complémentaires indépendantes du jeu qui ont pour vocation de rendre le volley-ball toujours plus spectaculaire et conforme au cahier des charges des medias audiovisuels.

\section{3. Les règles complémentaires indépendantes du jeu}

Dans cette nouvelle catégorie, il nous semble pertinent de dissocier deux domaines. D'une part, les règles dont la vocation est de réduire la durée de la rencontre. D'autre part les règles qui ont pour objectif de rendre le «produit » volley-ball conforme aux normes télévisuelles. Nous obtenons ainsi une représentation plus complète du règlement de volley-ball. 


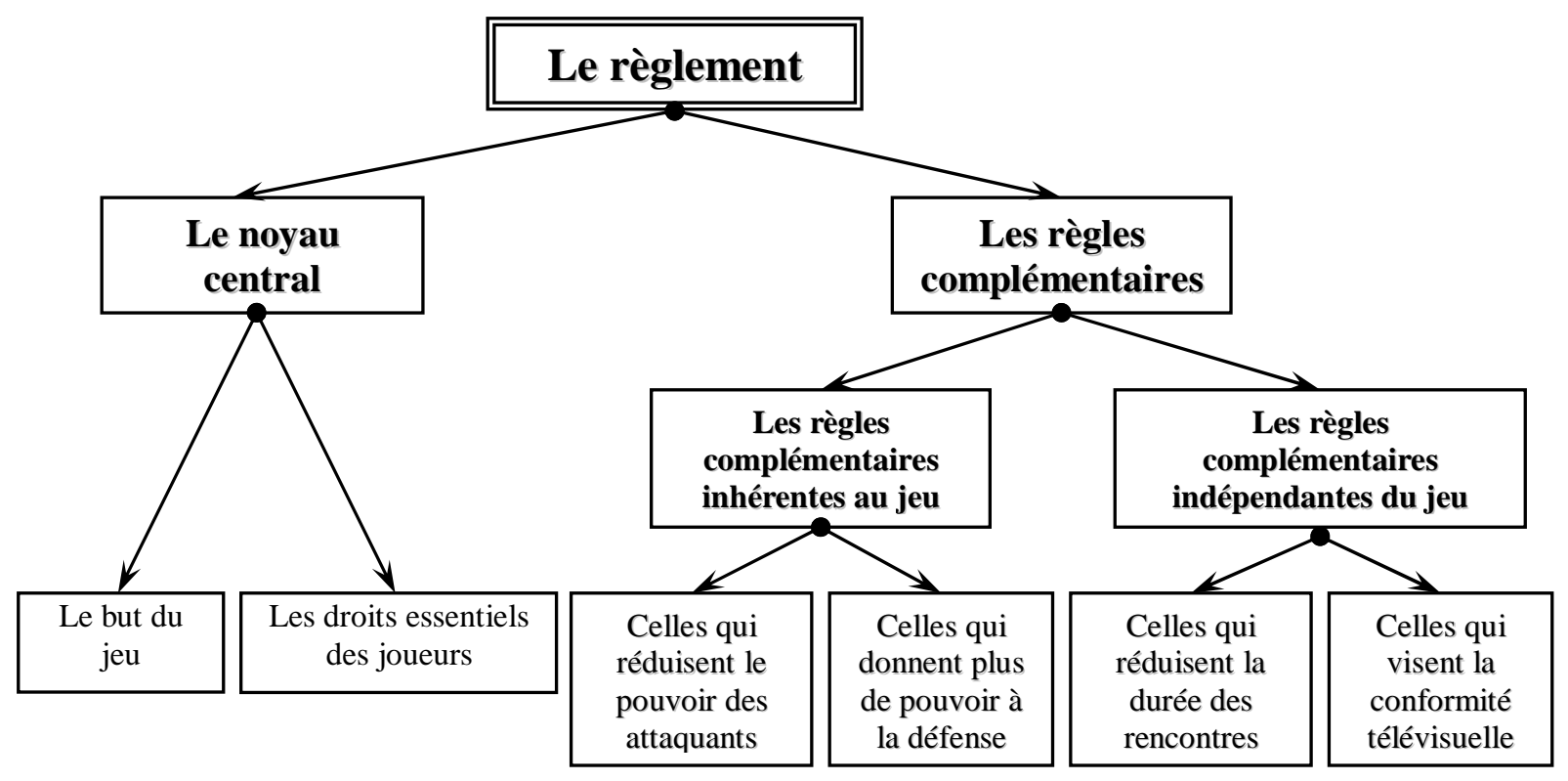

Figure 2: schéma de modélisation de la règle au volley-ball

À titre d'exemple nous allons rappeler quelques règles qui explicitent ce nouveau schéma.

3. 3. 1. Les règles indépendantes qui visent à réduire la durée de la rencontre

On a procédé tout d'abord à la mise en place de ramasseurs de balle qui ont eu pour mission de gérer 3 ballons (1978). Plus tard, pour le $5^{\mathrm{e}}$ set, il fut instauré le décompte des points dit en "marque continue » (1988). Cette décision n'a eu pour conséquence qu'une diminution de la durée des rencontres les plus disputées (et donc les plus longues) puisque seul le $5^{\mathrm{e}}$ set était concerné. Puis, comme nous l'avons évoqué plus haut, la marque continue fut appliquée à l'ensemble des sets (1999). Le score à atteindre est maintenant de 25 points pour les quatre premiers sets et de 15 point pour le $5^{\mathrm{e}}$ set.

3. 3. 2. Les règles indépendantes qui visent à rendre le "produit » volley-ball conforme aux normes télévisuelles

Bien évidemment, le fait de réduire le temps global des rencontres contribue à rendre les matchs de volley-ball plus diffusables. Cependant, cette conditions bien qu'étant indispensable n'est pas suffisante pour "attirer " les diffuseurs. Nous voyons au moins deux autres façon d'abonder dans le sens de la logique énoncée. La première consiste à s'ajuster aux normes de diffusion des autres programmes. II faut créer les conditions de rentabilisation des retransmissions. C'est la raison pour laquelle avec le nouveau décompte des points est apparue une nouvelle catégorie de temps morts. Ils sont automatiquement signalés par l'arbitre lorsque l'équipe qui mène atteint le score de 8 et 16 points (1999). Ces temps morts «techniques » ont une durée d'une minute. Pourquoi imposer de telles coupures qui vont augmenter la durée des sets ? La raison économique 


\section{eJRIEPS 23 avril 2011}

l'emporte ici sur toute autre considération. La possibilité de placer des spots publicitaires au beau milieu du jeu permet de «financer» les frais de diffusion. Bien sûr, les entraîneurs ont toujours à leur disposition deux temps morts de 30 secondes qu'ils peuvent prendre ou non en fonction des circonstances du jeu. Il est intéressant de noter que ce sont les temps morts «publicitaires » qui prennent le qualificatif de «temps morts techniques » dans le règlement.

La deuxième manière de valoriser la diffusion du volley-ball consiste à rendre toujours plus spectaculaire le jeu. C'est pour une part, dans cette optique que le rôle de «libéro » est apparu (1999). Il faut noter ici que la vocation première de ce joueur était de contribuer à renforcer la défense. Nous analyserons plus loin les véritables effets du libéro sur ce secteur de jeu. Quoi qu'il en soit, ce joueur est atypique puisqu'il « doit porter une tenue d'une couleur différente, contrastant nettement avec celle du reste de l'équipe » (règles FIVB 2009-2012, p.82). Mais au-delà du changement de tenue, quelles sont les conséquences de l'apparition de ce profil de joueur pour le scénario du match ? C'est ce que nous allons analyser maintenant.

\section{Le rôle de libéro}

La promesse de spectacularisation du jeu a été défendue par les plus hautes instances techniques du volley-ball. Dans son éditorial intitulé «La révolution par le spectacle » André Glaive alors Directeur Technique National pronostiquait : «Dans la foulée de l'apparition du libéro, voilà les trois sets gagnants en marque continue à 25 points. Ce n'est pas un changement, c'est une révolution : fini le volley-ball où le score restait parfois de longues minutes sans évoluer, lassant le spectateur et minant l'intérêt. Place à un spectacle vivant avec un score sans cesse en évolution, un libéro pouvant rentrer sans perdre de temps, un entraîneur pouvant se déplacer ! Les spectateurs seront ravis par ce nouveau jeu, simple à comprendre puisque chaque échange se concrétisera par un point mais également par sa durée limitée dans le temps. Les télévisions pourront plus facilement programmer une rencontre sans avoir à jongler avec le nombre de sets retransmis »(Glaive, 1998, p.2). Mais en quoi l'apparition du libéro devait-elle permettre d'augmenter le spectacle au volley-ball ? C'est le pari fait sur l'apport d'un joueur doté de compétences particulières en défense qui devait matérialiser les promesses de spectacularisation. «Spécialiser un expert de la défense visait à accroître le nombre de balles récupérées et mettre un terme à la succession aride de réception-passe-smash au profit d'échanges plus longs et plus spectaculaires » (Fournier, 2005, p. 127). L'examen du règlement nous explique pourquoi un tel joueur aurait pu être dédié à la défense. 


\section{eJRIEPS 23 avril 2011}

4. 1. Le libéro : droits et devoirs

Le règlement relatif au libéro tient en 2 pages (chapitre VI, p. 35 et 36) sur l'ensemble du règlement FIVB qui en compte 48 (FIVB, 2008).

Pour l'essentiel, le règlement nous dit :

- Le libéro peut remplacer n'importe quel joueur de la ligne arrière.

- II ne peut jouer que comme joueur arrière et n'est pas autorisé à effectuer une frappe d'attaque d'où que ce soit (y compris le terrain de jeu et la zone libre) si, au moment du contact, le ballon est entièrement plus haut que le bord supérieur du filet.

- II ne peut ni servir, ni contrer ou tenter de contrer.

- Un ballon provenant d'une touche haute jouée avec les doigts et effectuée par un libéro dans sa zone avant ne peut être attaqué plus haut que le bord supérieur du filet. Le ballon peut être attaqué librement si le libéro effectue la même action en dehors de sa zone.

Pour résumer :

- Le libéro est un arrière qui ne peut ni attaquer, ni jouer le rôle de passeur, ni servir.

- Il peut réceptionner et défendre.

Fait particulier, alors que tous les changements de joueurs doivent être inscrits sur la feuille de match, le libéro rentre et sort librement si ces changements se font entre deux échanges. Mais qui va-t-il remplacer?

4. 2. Le libéro : aspects stratégiques

Pour répondre à cette question, il nous faut munir le lecteur de quelques connaissances supplémentaires. Car la question de l'utilisation du libéro est intimement liée à la question du joueur qu'il va remplacer. Les problématiques stratégiques sont donc centrales pour rendre compatibles les exigences réglementaires et les bénéfices qu'un tel profil de joueur peut apporter à l'équipe.

\section{2. 1. La spécialisation en volley-ball}

Depuis la naissance du volley-ball, nous sommes passés d'un universalisme prescrit à une spécialisation inéluctable. L'obligation règlementaire des rotations de l'équipe au service (la rotation du serveur devient obligatoire en 1916), fait apparaître un universalisme apparemment structurel. Cette affirmation peut être corroborée par le fait que les joueurs interviennent à tour de rôle à tous les postes d'avants comme d'arrières pour y accomplir successivement toutes les actions y compris celle de servir. De ce fait, l'idée d'universalisme peut être définie comme le principe d'organisation collective selon lequel tous les joueurs sont amenés à jouer tous les rôles en fonction des circonstances du jeu. Mais il s'avère que le développement stratégico-tactique du volley-ball a suivi une voie que d'autres activités humaines ont également empruntée. Comme le suggèrent 


\section{eJRIEPS 23 avril 2011}

certaines conceptions de l'organisation du travail (le Taylorisme notamment), l'activité peut-être découpée en différentes tâches. La rentabilité de l'équipe passe alors par l'augmentation de la performance des joueurs dans des tâches particulièrement ciblées. L'idée de spécialisation a alors conduit à la mise en place d'une organisation collective dans laquelle chaque joueur a un rôle précisément défini indépendamment des circonstances du jeu. Cela a conduit à une répartition des tâches en fonction des différents postes. Cette évolution fut encadrée réglementairement par les dispositions relatives à la formation des équipes. Un ensemble de règles permet de définir ce qu'est une faute de position. II serait fastidieux ici de faire une description exhaustive de ces points de règlement. Nous donnerons néanmoins deux règles essentielles qui éclairent cette notion. "La fiche de position indique l'ordre de rotation des joueurs sur le terrain. Cet ordre doit être respecté pendant la totalité du set « (Règle 7.3.1. b. Règlement FIVB). La fiche de rotation remise par l'entraîneur à l'arbitre avant chaque set indique l'ordre de service pour la totalité du set. On tourne au service sans jamais omettre le tour de l'un des joueurs. II y a donc assurément un intérêt à choisir qui sera le premier serveur. Peut-être pour sa qualité de service mais aussi pour le choix de la position de son équipe. «Au moment où le ballon est frappé par le joueur au service, chaque équipe doit être placée dans son propre camp (excepté le joueur au service), selon l'ordre de rotation » (Règle 7.4. Règlement FIVB). Ces positions doivent être respectées jusqu'à la frappe de balle du serveur. Ensuite, chaque joueur peut occuper sur le terrain la position qui lui convient. Cependant, arrières et avants conservent leurs prérogatives.

4. 2. 2. Les différents profils de postes et composition d'une équipe

Puisque le libéro peut remplacer n'importe quel joueur de la ligne arrière, quel joueur va-til remplacer? La réponse à cette question nécessite de se représenter la composition d'une équipe c'est à dire, la répartition des postes les uns par rapport aux autres (puisque l'équipe est amenée à tourner). Pour le haut niveau, il existe actuellement cinq profils de postes différents.

- Le passeur : c'est le distributeur et le tacticien offensif de l'équipe. Celui par qui tous les ballons passent (Éloi, 2007 ; 2009).

- Le pointu : c'est l'attaquant type, le frappeur. Celui à qui l'on donne le ballon pour se sortir des situations les plus délicates.

- Les réceptionneurs attaquants : ils sont deux et comme leur nom l'indique, ils jouent un rôle important lors de la réception (récupération du service adverse) et l'attaque. 


\section{eJRIEPS 23 avril 2011}

- Les contreurs centraux: ils sont spécialisés dans les attaques de premiers temps (attaque rapide) et sont les pièces maîtresses du contre. Ils se positionnent au milieu du filet et tentent d'intercepter les smashs adverses.

- Le libéro : il réceptionne et défend et ne peut être qu'arrière.

Du fait que les équipes sont alternativement en rotation, il est nécessaire d'équilibrer les placements des joueurs les uns par rapport aux autres. II s'agit donc de positionner sur chaque ligne (avant et arrière) des profils de postes équivalents. L'évolution historique des organisations strategico-tactiques a conduit à répartir les spécialités de la façon suivante. Ceci est le schéma le plus répandu actuellement.

On observe que contreurs centraux et réceptionneurs-attaquants sont placés en opposition tout comme le passeur et le pointu.

4. 2. 3. Quel joueur le libéro remplace-il ?

L'apparition des services smashés requiert la présence de 3 réceptionneurs. II est donc exclu de faire sortir un réceptionneur-attaquant. Le passeur distribue le jeu. II est donc indispensable lors de toutes les phases de jeu. Idem pour le pointu qui peut être sollicité à tout moment. II n'y a donc que les contreurs centraux dont la présence ne soit pas indispensable sur la ligne arrière. Le libéro va donc (dans la grande majorité des cas) remplacer alternativement chacun des deux contreurs centraux (après son service puisque le libéro ne peut pas servir).

4. 2. 4. Organisation du rapport d'opposition en volley-ball

II est communément admis que le rapport de force en volley-ball est constitué de 6 oppositions. II y a 6 joueurs, donc 6 rotations, donc 6 confrontations à l'adversaire. II nous semble qu'il faut approfondir la réflexion si l'on veut véritablement analyser le rapport de force dans notre discipline. En effet, s'il est vrai que les deux équipes occupent 6 positions successives, il ne faut pas occulter que ces rotations se font alternativement. Comme le montre le schéma suivant, un set est donc la succession de 12 oppositions différentes qui se présentent cycliquement. Un set correspond environ à 2,5 tours ${ }^{2}$ pour chaque équipe (Albert, 2010).

\footnotetext{
${ }^{2}$ Une étude d'Albert (2010), montre que sur 21 matchs (matchs retours et play-off) du Tours Volley-Ball (champion de France 2009-2010), la moyenne est de 2,54 rotations si l'on ne prend en compte que les sets qui se disputent en 25 points. Cette moyenne descend à 2,51 si l'on prend en compte tous les sets y compris les tie-breaks qui se jouent en 15 points.
} 

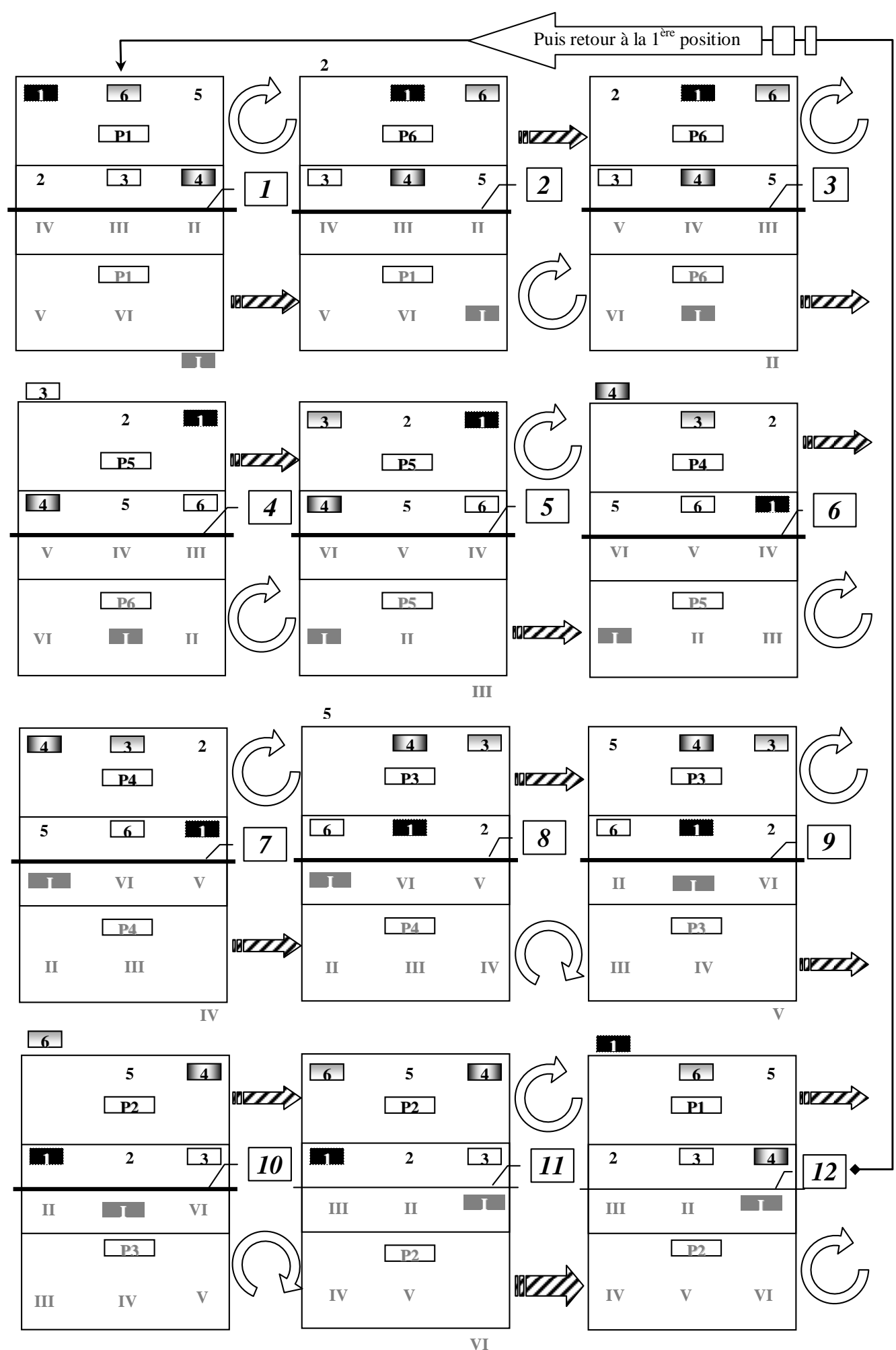

Figure 4 : un set est la succession de 12 oppositions différentes qui se présentent cycliquement.

\section{Passeur \\ 4 Pointu \\ 3 Libéro \\ 6 Contreur central 5 \& 2 Réceptionneurs attaquants}

Grace à cette représentation, on peut compter le nombre de position de chacun des joueurs quand l'équipe est au service ou en réception. 
4. 2. 5. Effets engendrés par l'utilisation du libéro sur le rapport attaque-défense Nous l'avons précédemment souligné, le libéro ne peut pour ainsi dire que réceptionner et défendre. Dans le calcul du rapport attaque-défense il est nécessaire d'assimiler qu'augmenter la capacité à réceptionner engendre une amélioration de l'attaque (Cf. chapitre 2.3.). Mais le libéro est aussi un défenseur. II peut donc renforcer l'aptitude à défendre d'une équipe. La représentation est encore solidement ancrée que le libéro augmente le potentiel défensif. Nous nous proposons maintenant de faire un bilan comptable des situations de réception (et donc d'attaque) et des situations de défense du libéro. Prenons le cas de l'équipe qui se trouve sur la partie supérieur du terrain (figure 4). En observant ce schéma, on constate que sur un total de 12 oppositions, le libéro réceptionne sur 6 positions (oppositions 1, 3, 5, 7, 9 et 11) alors qu'il ne défend que sur 4 positions (oppositions 2, 6, 8 et 12). En effet, le libéro sort sur 2 positions de défense (lorsque sont équipe est au service) puisque chaque contreur central doit servir (opposition 4 et 10). On peut donc conclure mathématiquement que le libéro renforce plus l'attaque qu'il ne consolide sa défense. Ainsi, l'introduction de ce nouveau profil de poste n'a pas eu les conséquences annoncées lors de son intronisation. Quelles sont les circonstances qui ont abouti à cette «innovation ». À l'origine, ce sont les pays asiatiques qui proposèrent l'introduction d'un joueur spécialisé en défense. L'une des caractéristiques des joueurs asiatiques est qu'ils sont très vifs et adroits en défense. Mais cette proposition imposait que ce joueur ne soit sur le terrain que pendant les 6 phases de service, c'est à dire uniquement pour défendre. Or, deux de ces phases nécessitaient la présence des contreurs centraux pour servir. Dans la proposition initiale, le libéro ne pouvait donc rester sur le terrain que lors de 4 oppositions sur 12 sur le terrain ce qui était insuffisant pour justifier la création d'un nouveau rôle. Après beaucoup de discussions et d'expérimentations, un compromis a abouti à la naissance du libéro tel qu'il est aujourd'hui. Mais le bilan actuel démontre que ce joueur participe notoirement à un renforcement de l'attaque. Ce constat est donc en totale contradiction non seulement, avec l'esprit de la proposition asiatique, mais aussi avec le développement historique de la règle du volley-ball.

\section{Quand "rapport attaque-défense " et «spectacularisation" entrent en contradiction}

Nous l'avons déjà signalé précédemment, les règles complémentaires indépendantes du jeu et les règles complémentaires inhérentes au jeu finissent par rentrer en contradiction. La mise en place du libéro sous prétexte de spectacularisation abouti en fait au résultat 


\section{eJRIEPS 23 avril 2011}

inverse de celui qui était escompté. Mais d'autres effets collatéraux sont maintenant palpables.

\section{1. Stratégies induites}

En effet, la disproportion entre le pouvoir de l'attaque et celui de la défense conduit à développer des stratégies qui orientent l'évolution du jeu lui-même.

5. 1. 1 Assurer «le side out » (phases de réception)

Puisque la difficulté à défendre devient considérable, la stratégie des entraineurs consiste à privilégier la phase de service-réception. Pour cela, l'accent est mis lors de l'entraînement sur la répétition de combinaisons offensives prédéterminées et sur son corollaire, le développement d'une réception très performante. Dans cette optique, le libéro qui a une double valence (réceptionneur et défenseur) voit son aptitude à réceptionner particulièrement valorisée. Et de fait, l'entraînement renforce l'habileté du libéro en réception souvent au détriment de sa capacité à défendre.

5. 1. 2. Tenter le tout pour le tout sur phase de service

Par ailleurs, puisque les équipes sont devenues extrêmement performantes sur phase de réception, la stratégie du serveur consiste à tenter le tout pour le tout. Les statistiques montrent que l'équipe au service a 1 chance sur 3 de gagner l'échange. Le serveur est donc encouragé à prendre des risques considérables pour tenter de détruire la réception adverse. Cela implique un service très puissant (donc très risqué) pour réduire la possibilité de distribuer le jeu avec tous les choix possibles pour le passeur adverse. L'idée est qu'un tel service doit diminuer les choix d'attaques chez l'adversaire. Un contre bien organisé peut alors prendre le pas dans ces situations où les choix d'attaques sont restreints et l'incertitude réduite (puisqu'il y a diminution des alternatives d'attaques).

\section{2. Conséquences sur l'évolution du jeu}

Cette analyse du jeu est très répandue dans le milieu du volley-ball (propos recueillis lors des formations professionnelles continues des entraîneurs de la ligue nationale de volleyball). Elle conduit à expliquer le spectacle qu'il est donné de voir régulièrement.

5. 2. 1. Un spectacle sous influence

Le jeu se résume donc à une succession d'échanges dans lesquels la balle ne passe que deux fois le filet. La première fois correspond au passage du service ; la seconde, à l'attaque de l'équipe en réception. Si la réception est réussie (à haut niveau, le pourcentage de réception qui permet au passeur de choisir entre tous ses attaquants est 


\section{eJRIEPS 23 avril 2011}

de $65 \%^{3}$ ). On constate que dans ces situations, les 3 contreurs doivent s'opposer à 4 attaquants (avec 1 ou 2 attaquants aux 3 mètres) ce qui laisse peu de chance à la défense. Le second passage du filet est donc souvent le dernier de l'échange. Mais il n'est pas rare d'observer une succession d'échecs au service (même si l'on peut observer un retour du service flottant rendu plus ${ }^{4}$ efficace de par la nouvelle texture du ballon). D'une façon générale, la prise de risque est telle que l'on peut assister à des séries de mises en jeu où les serveurs de chaque équipe se succèdent sans que le spectacle ne débute réellement. On comprend que le public « s'enflamme » dès que l'échange dure un peu. Gibout et Mauny nous invitent à faire le lien entre la performance et le spectacle. «La performance se conçoit dans l'atteinte d'un résultat brut, d'un avantage au tableau d'affichage. Le plaisir se conçoit dans la forme gestuelle, le sentiment de liberté et dans la maîtrise intellectuelle de la situation. Si la performance et le plaisir génèrent des émotions de nature différente, l'esthétique et la créativité gestuelles de l'expert sont génératrices d'émotions plus intenses lorsqu'elles suscitent du spectacle, du spectaculaire, de l'orgasmique »(Gibout et Mauny, 2006, p.154). Dans ces exceptionnels rallyes où les actions défensives sont souvent étonnantes, le côté spectaculaire du volley-ball se révèle laissant alors entrevoir les potentialités d'un divertissement remarquable.

\section{2. 2. Influence sur le recrutement}

Du fait que les phases de réception sont survalorisées, la valence principale du libéro 5 évolue radicalement vers celle de réceptionneur. Les entraîneurs recherchent donc au moment de leur recrutement des libéros qui ont plutôt ce profil. Les libéros connaissant «les lois du marché » sont donc particulièrement motivés pour développer leurs compétences de réceptionneur. Le risque d'un tel engrenage pourrait aboutir à terme, à la condamnation des libéros à valence « défensive ».

\section{2. 3. La valence du libéro comme facteur aggravant}

Le profil du poste risque donc en retour de contribuer à l'accroissement et à la pérennité du déséquilibre attaque-défense. Le jeu en vaut-il la chandelle ? II semble alors légitime de se demander en quoi le libero renforce le côté spectaculaire du volley ? Car une réception est rarement spectaculaire.

\section{Pour sortir de l'impasse}

\footnotetext{
${ }^{3}$ Ce pourcentage est devenu une norme. Ces données issues de nos propres statistiques portent sur des équipes de Ligue $A$ masculines. Elles ont été réalisées pour chaque match, lors des 10 dernières saisons, dans le cadre de notre activité d'entraîneur.

${ }^{4}$ Cette tendance ne pourra être confirmée que dans 1 ou 2 saisons. Le temps nécessaire pour les réceptionneurs de s'adapter ou non.

${ }^{5}$ Informations recueillies auprès des autres entraineurs et des agents de joueurs.
} 


\section{eJRIEPS 23 avril 2011}

Le volley-ball souffre d'un déficit chronique du pouvoir de la défense sur l'attaque. De plus, la mise en place du libéro semble donner un coup d'arrêt au développement des ambitions défensives chez les techniciens. L'argumentation reste pragmatique. II est utopique de vouloir défendre, donc il est préférable de renforcer le potentiel offensif. Mais plus le potentiel offensif se développe et moins il est possible de défendre. Des propositions de changement des lois du jeu doivent permettre de sortir de ce cercle vicieux.

\section{1. Proposition de changement de règle}

Quelles ont les possibilités qui s'offrent au législateur? On peut évidemment changer les règles indépendantes du jeu. II nous semble que cela reviendrait à mettre un pansement sur une jambe de bois. La présence des «pom-pom girls» sur le terrain pendant les temps morts techniques n'améliore pas le spectacle du jeu en lui-même. La solution se trouve donc certainement du côté des règles inhérentes au jeu. Ce qui rend le spectacle « volley-ball » attrayant est contenu dans l'incertitude de l'issue de l'échange. D'une part, il faut rétablir l'équilibre entre l'équipe au service et l'équipe en réception. «Plus que la durée de l'échange, l'évaluation du nombre de passages du ballon au dessus du filet semble être le bon choix » (Blain, 2010). II faut donc sortir de la routine des 2 passages de balle observée actuellement. D'autre part, il faut envisager qu'une incertitude soit maintenue dans le nombre de ces passages. Organiser le suspens c'est faire en sorte que les échanges avec 1, 2, 3 ou plus de passages de ballon soient observés à part égale. II reste donc deux solutions. La première consiste à augmenter les droits des défenseurs. II nous semble que jusqu'à maintenant, tous les efforts ont été concentrés dans cette direction. Accentuer encore les droits de la défense ne peut aboutir qu'à augmenter la tolérance faite sur les touches de balle. Cette solution n'est pas envisageable car elle rentrerait en contradiction avec le fait qu'au volley-ball, la balle doit être frappée. II faut donc s'attaquer aux droits de l'équipe à l'attaque. Or il s'avère que le surnombre des possibilités offensives est obtenu par la possibilité des attaques aux 3 mètres. Une solution intéressante pourrait concerner la diminution du potentiel offensif des arrières. Nous proposons de reculer la ligne des 3 mètres pour la placer à 4,50 mètres. On séparerait donc en deux chaque côté du terrain. Zone arrière et zone avant auraient alors la même surface. En obligeant les attaquants arrières à smasher en prenant leur appel derrière cette nouvelle démarcation, on réduirait drastiquement l'efficacité de ces attaques sans porter atteinte au côté spectaculaire de ces tentatives. La hauteur de frappe, leur synchronisation avec les attaques des avants, la puissance des frappes seraient conservées. Mais du coup, au lieu d'attaquer à un mètre du filet (voire moins), on peut 


\section{eJRIEPS 23 avril 2011}

estimer que les attaquants arrières smasheraient à environ 2,5 mètres. On ne ferait d'ailleurs que remettre au goût du jour la règle de 1922 qui avait introduit la ligne des 3 mètres comme marque de séparation des avants et des arrières. Obliger les arrières à attaquer plus loin du filet ne fait que prendre en compte les qualités physiques actuelles pour retrouver l'équilibre qui avait été recherché il y a 89 ans.

On peut relever un inconvénient à cette proposition. Celui de devoir retracer les lignes actuellement placées à 3 mètres du filet. Les bandes adhésives pourront pallier momentanément à cette difficulté en attendant que la peinture ne marque définitivement cette nouvelle démarcation.

Un certains nombre de règle resteraient inchangés :

- 13.2. $2^{6}$ Un arrière peut effectuer une attaque à n'importe quelle hauteur en arrière de la zone avant ;

- 13.2.2.1 lors de son impulsion, son (ses) pied(s) ne doit (doivent) avoir ni touché ni franchi la ligne d'attaque;

- 13.2.2.2 après la frappe, il peut retomber dans la zone avant.

\section{Une seule règle devrait donc subir une modification :}

\section{- 1.3.4 Ligne d'attaque}

Dans chaque camp, une ligne d'attaque, dont le bord extérieur est tracé à $3 \mathrm{~m}$ de l'axe de la ligne centrale, délimite la zone avant (règle actuelle)

Cette règle actuelle serait changée ainsi :

\section{- 1.3.4 Ligne d'attaque}

Dans chaque camp, une ligne d'attaque, dont le bord extérieur est tracé à 4,5 m de l'axe de la ligne centrale, délimite la zone avant (nouvelle règle)

Les autres règles qui dépendent de cet article n'auraient pas à être modifiées.

\section{2. Conséquences pour le rapport d'opposition}

Tentons maintenant d'anticiper les effets de ces nouvelles dispositions. Pour les contreurs, seuls les joueurs adverses de la ligne avant deviennent des menaces de premier plan. Les contreurs peuvent se focaliser sur les attaques au filet. Le rapport de force au filet est donc rétabli. Sur 3 positions (le passeur est arrière), les 3 contreurs s'opposent à 3 attaquants de la ligne avant. Sur les 3 autres (le passeur est avant), à 2 attaquants seulement. Pour la défense basse, les attaques depuis la zone arrière deviennent contrôlables. Avec une augmentation d'au moins 1,5 m des trajectoires d'attaque, l'impact du ballon sur le défenseur est significativement réduit. Mais une autre conséquence

\footnotetext{
${ }^{6}$ Ces chiffres correspondent aux numéros des articles du règlement.
} 


\section{eJRIEPS 23 avril 2011}

concerne l'espace que l'attaquant de la ligne arrière peut alors atteindre. En effet, comme le montre le schéma ci-dessous, en reculant l'impact du frappeur (à hauteur de frappe identique), l'angle possible d'attaque possible se réduit considérablement. La surface à défendre est donc moins importante. Dans ces conditions, les défenses deviennent à nouveau possibles.

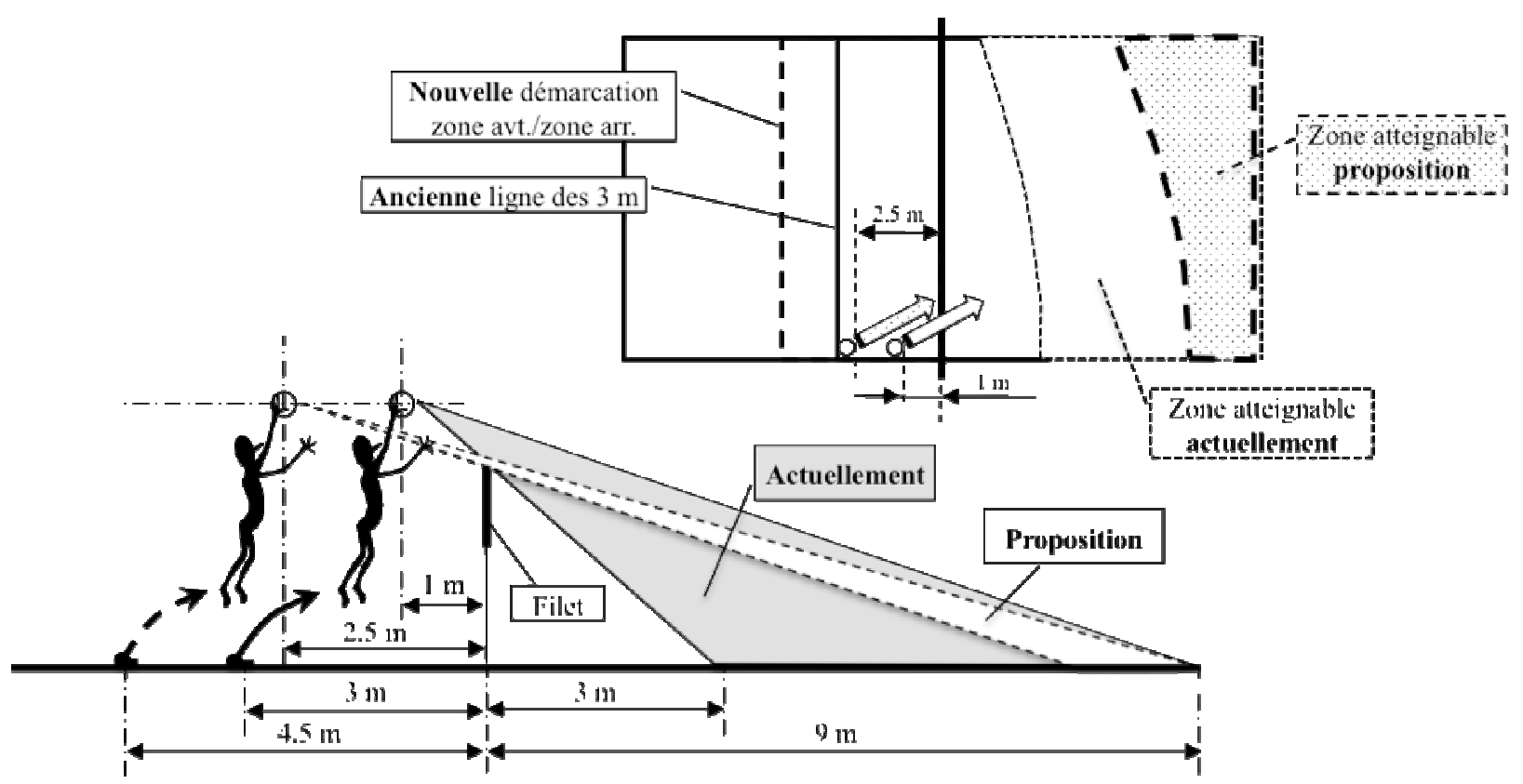

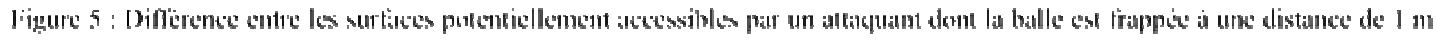

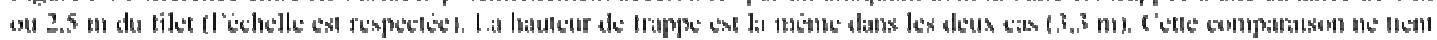

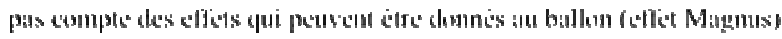

La présence d'un libéro défenseur constitue alors un véritable atout. Et du coup, c'est une bascule possible vers le développement de la défense qui s'opère.

\section{Réflexion sur la démarche technologique}

Dans le but de discuter de la démarche technologique, c'est sur l'analyse de la méthode que nous voulons revenir maintenant. La proposition de modification du règlement décrite plus haut sera soumise à la commission des lois du jeu de la FIVB. Mais au-delà de la proposition elle-même, c'est la démarche et l'argumentation développée qui nous paraissent relever d'une démarche technologique. En procédant maintenant à cette «méta-analyse ${ }^{7}$, il s'agit de porter un regard sur les instruments mis en jeu dans la désintrication et la compréhension des différents facteurs du développement du volley-ball en tant que pratique physique socialement partagée. Partagée de l'intérieur, par les

\footnotetext{
${ }^{7}$ Méta-analyse : il s'agit de mener ici une analyse de l'analyse.
} 


\section{eJRIEPS 23 avril 2011}

pratiquants qui vivent leur activité physique favorite. Partagée de l'extérieur, par les spectateurs à qui l'on se doit de proposer un divertissement attrayant.

\section{1. Les registres de technicité}

Nous avons évoqué la référence à un registre principal : le registre de participation. En effet, notre réflexion n'avait pas pour but d'analyser précisément le jeu pour lui-même afin d'en tirer des enseignements immédiats pour l'entraînement ou la préparation d'une rencontre. Elle a au contraire nécessité de prendre du recul. Un recul historique pour dévoiler les tendances de développement de la règle. Un recul affectif pour atteindre à une certaine rationalité. Ce n'est pas l'entraîneur ou le joueur qui réagit. C'est l'investigateur qui en adoptant une posture anthropologique prend la mesure de la complexité des faits. Et parmi ces faits doivent être pris en considération ceux qui sont relatifs aux registres associés: le registre de maîtrise et le registre de transformation. D'une part, le registre de maîtrise car l'évolution de la règle n'est pas sans conséquences sur les aspects stratégico-tactiques et tactico-techniques. La règle induit d'adapter la relation à l'adversaire et par là même d'ajuster les techniques produites. Et de fait, les changements successifs dans l'arbitrage de la $1^{\text {ère }}$ touche de balle de réception ont conduit à réceptionner à 2 mains hautes les services flottants. Les réceptionneurs ont donc pu (dû) se positionner plus près du filet. D'autre part, cette étude à également alimenté le registre de transformation car comme nous l'avons vu plus avant, le rapport attaque-défense existant oriente la valence des libéros. Ainsi, l'évolution de la règle conduit à modifier le rapport de force. Ce rapport détermine des stratégies globales (minorer la défense au profit de la phase de réception). Ces stratégies conduisent alors à orienter le profil du libéro qui est devenu indubitablement et principalement un réceptionneur. Les vœux de spectacularisation ne peuvent pas être sans conséquence sur les aspects stratégiques, tactiques ou techniques de notre discipline. Pour Vigarello, « c'est bien ce nouveau mode de rassemblement festif, cette mise en scène d'une liturgie laïque, qui a eu une influence sur les pratiques, autant que sur les techniques ellesmêmes. Rendre les pratiques plus spectaculaires, c'est souvent transformer les techniques qui les sous-tendent »(Vigarello, 1988, p. 147).

\section{2. Des artefacts dédiés à l'analyse de la règle}

Pour mettre à jour les logiques profondes qui permettent de développer la règle, nous avons dû construire différents artefacts. Ces artefacts sont en fait des outils chargés d'une dimension conceptuelle.

- Des artefacts cognitifs: ils nous ont permis d'expliciter l'organisation générale de la règle. Ils ont parfois pris la forme d'organigrammes. En effet, ce mode de représentation (figures 


\section{eJRIEPS 23 avril 2011}

1 \& 2) permet de mettre en œuvre une relation ordonnée entre les éléments. Et c'est au travers de ces relations que la référence à du conceptuel s'opère.

- Des artefacts matériels : de par leur fonction représentationnelle, les schémas et les dessins techniques (en opposition au dessin d'art) révèlent une réalité invisible au premier abord. Le schéma permet de diffuser de la connaissance (figure 3). Mais il peut aussi organiser les données disponibles pour les faire parler (figure 4). Ici encore, il devient possible de faire apparaître les éléments cachés d'une réalité. Le dessin technique quant à lui permet de vérifier qu'une hypothèse est recevable. A l'instar de la résolution graphique d'un problème de mécanique, le dessin technique s'apparente à un modèle. En permettant de moduler les données initiales du problème il devient possible d'en déterminer les conséquences (figure 5). Ainsi, le dessin technique rend compte des effets vraisemblables d'une situation quand il serait fastidieux de la mettre en œuvre dans la réalité.

- Un système d'instruments: plus que des outils dispersés, ces différents artefacts constituent un véritable système d'instruments. D'une part, ils passent du statut d'artefact à celui d'instrument au moment même où s'opère le basculement de la description de la réalité à la découverte de la logique qui l'organise. Cette découverte marquant réellement l'appropriation de l'outil par celui qui l'utilise dans un processus d'instrumentalisation (Rabardel, 1995). D'autre part, l'ensemble de ces instruments forment un système c'est à dire, un ensemble d'éléments qui ne prend sens que dans le rapport de ses éléments les un avec les autres. «C'est la logique de notre activité située, concrète et singulière qui, dans ce cas, a organisé les relations de complémentarité fonctionnelle entre les instruments et les séquences temporelles de leurs usages successifs ou concomitants » (Rabardel \& Bourmaud, 2009, p. 211).

\section{3. Un long processus : la genèse instrumentale}

Mais il faut du temps pour construire de tels outils. II a d'abord fallu tâtonner, établir des schémas, les modifier, les faire évoluer pour qu'ils rendent compte de la réalité. Puis en prenant du recul, il a été possible de poser des questions. Pour y répondre il a fallu parfois faire évoluer les outils. II y a donc dans la genèse instrumentale un double mouvement. Tout d'abord, la construction des artefacts qui nécessite l'utilisation, la transformation, le développement des représentations du problème. D'autres part, leur appropriation, leur transformation, voire leur détournement. L'exemple de la figure 4 est édifiant à cet égard. Au début des années 2000, nous avons construit un logiciel qui permettait de positionner 


\section{eJRIEPS 23 avril 2011}

notre équipe en fonction de la position de l'équipe adverse ${ }^{8}$. II existait bien des outils « papier-crayon » mais qui étaient peu modulables et non utilisables dans un court laps de temps (il n'y a que 3 minutes entre 2 sets et il faut changer de côté). Avec l'informatique, un simple clic sur un bouton permet de faire tourner les équipes. On peut alors immédiatement observer les conséquences de ces nouvelles oppositions de lignes. C'est donc à cette occasion que nous avons pu poser que le volley-ball est une succession de 12 oppositions (et non 6 comme on peut l'entendre couramment). L'utilisation de ce schéma dans le cadre de l'analyse ici développée relève donc d'une catachrèse. II y a bien détournement du schéma initial pour en faire un usage divergent. C'est à cette condition qu'il est devenu possible de déterminer l'influence du libéro sur le rapport d'opposition. En détournant l'instrument initial de sa fonction première, il nous a été possible de lui faire remplir une autre mission dans laquelle il s'est actualisé. Ainsi donc, «l'instrument incorpore, sous ses formes spécifiques, les rapports fonctionnels et subjectifs aux objets de travail, à soi-même ici et dans le futur, aux autres, aux collectivités et à la culture de la société dans laquelle le sujet s'inscrit »(Rabardel, 2009, p.18).

\section{Conclusion : de l'analyse technologique à un test grandeur nature}

Nous avons choisi d'emprunter une démarche technologique. Cela nous a notamment aidé dans la construction d'un modèle de la règle au volley-ball. Nous avons pu ainsi mettre en évidence une contradiction entre la volonté historique de donner toujours plus de pouvoir à la défense (dans le but de rééquilibrer le rapport de force attaque/défense) et les velléités nouvelles (1999) de spectacularisation soutenues par les dirigeants des instances internationales. L'imbrication des registres et des artefacts nous a permis d'appréhender la complexité sous un nouveau jour. II a même fallu parfois détourner les instruments originaux de leur fonction première dans le but de comprendre une situation nouvelle. N'est-ce pas là le propre de la démarche technologique. Cette structuration des différents éléments en un modèle logique nous a permis d'envisager qu'une solution probable à l'impasse dans laquelle nous nous trouvons actuellement pourrait résider dans une diminution drastique des pouvoirs de l'attaque. En imaginant que la commission des lois du jeu de la FIVB fasse connaître son intérêt pour la proposition de changement de règle que nous avons exposé précédemment, il est assez peu probable que cette règle puisse être appliquée immédiatement. II faudra pour justifier la mise en place de ce changement que des tests grandeur nature soient effectués. II nous semble que la

\footnotetext{
${ }^{8}$ Vendu alors pour $1 €$ symbolique à tous les entraîneurs de Pro $A$ et bien d'autres, ce logiciel est toujours utilisé.
} 


\section{eJRIEPS 23 avril 2011}

pratique universitaire pourrait être un excellent terrain de mise à l'essai. En effet, cette compétition concerne autant les garçons et les filles. De plus, elle permet de confronter tous les niveaux de jeu (du débutant à l'expert). Enfin, elle se déroule sur une saison entière avec des phases finales qui regroupent les meilleurs niveaux. En se prêtant à cette « expérimentation », le volley-ball universitaire pourrait trouver un autre intérêt que celui de la simple réplique du sport fédéral. Cette pratique distincte donnerait un autre sens à la participation étudiante aux activités de la Fédération Française du Sport Universitaire. Elle contribuerait dans le même temps aux missions de recherche et d'innovation de l'Université.

\section{Bibliographie}

Albert, N. (2010). La position de départ d'une équipe de volley-ball en début de set : le choix de l'entraîneur. Mémoire de Master 2 - Entraînement du sportif de haut niveau - Parcours international en volley-ball. Université Paris Est - Créteil.

Blain, P. (2010). Conclusions au sujet de la Golden Formula. Volley France Tech, revue de la Direction technique Nationale de la FFVB. Numéro 28 - mars 2010.

David (1993). Place et rôle des représentations dans la mise en œuvre didactique d'une APS : l'exemple du rugby. Thèse de doctorat non publiée, Université Paris XI.

Coulon, A. (1991). L'usage de la règle dans la pratique sportive. In J. Ardoino \& J.M. Brohm (Eds). Anthropologie du sport. Perspectives critiques. Actes du colloque international francophone. (pp. 185-190). Paris-Sorbonne, 19-20 avril 1991. Andsha - Matrice - Quel Corps ?

Demiselle, J. (2005). Enseigner à partir de l'évolution historique du rapport de force attaque-défense en volley-ball. In J-M. Legras (Eds.). Vers une technologie culturelle des APSA. (pp. 231-254). Paris : Vigot.

Deplace; R. (1979). Rugby de mouvement rugby total. Paris : Ed. EPS.

Deleplace, R. (1983). La recherche sur la spécialisation sportive, l'entraînement, la performance. Actes du colloque «la recherche en STAPS». (pp. 93-151). Nice 19-20 septembre 1983.

Durey, A. \& Bouthier, D. (1994). Technologie des activités physiques et sportives. Impulsions, 1, 117-126.

Éloi, S. (1999). Acte du colloque des entraîneurs de Nantes - Ligue mondiale de volleyball. Samedi 26 et dimanche 27 juin 1999. 


\section{eJRIEPS 23 avril 2011}

Éloi, S. (2007). Rapport de recherche FFVB -MJS - Université Paris Est Créteil. Identification des compétences requises par les passeurs de haut niveau en volley-ball. $302 \mathrm{p}$.

Éloi, S. (2009). Style d'un passeur de haut niveau en volley-ball. eJRIEPS, 17, 76-105

Éloi, S. et Ulrich, G. (2001). Contribution à la caractérisation des sports collectifs : les exemples du volley-ball et du rugby. STAPS, 56, 109-125.

FIVB (2008). Règles officielles du volley-ball 2009-2012. Approuvées par le 31ème Congrès de la FIVB 2008. Fédération Internationale de Volley-Ball.

Fournier, P. (2005). Modification de la codification d'un sport et son impact sur le jeu : l'exemple de la règle du libéro en volley-ball. Science \& Motricité, 56, 125-140.

Gibout, C. \& Mauny, C. (2005). Les représentations symboliques comme moyen de redéfinition de ré-appropriation des techniques corporelles en sports collectifs. In L. Robène, \& Y.Léziart (Eds). L'homme en mouvement, vol. 2. (pp. 147-171). Paris : Chiron.

Glaive, A. (1998). La révolution par le spectacle. Volley France Tech, revue de la Direction technique Nationale de la FFVB. Numéro 3 - novembre 1998.

Jeu, B. (1983). Le sport, l'émotion, l'espace. Paris, Vigot.

Le Moigne, J.-L. (1999). La modélisation des systèmes complexes. (2ed.). Paris : Dunod.

Martinand, J.L. (1994). La didactique des sciences et de la technologie et la formation des enseignants. ASTER, 19, 61-75.

Pastré, P. (1999). La conceptualisation dans l'action: bilan et nouvelles perspectives. Éducation permanente, 139, 13-35.

Rabardel, P. (1995). Les hommes et les technologies. Approche cognitive des instruments contemporains. Armand Colin. 239 p.

Rabardel, P. \& Bourmaud, G. (2009). Instruments et système d'instruments. In Rabardel, P. \& Pastré, P. (Eds). Modèles du sujet pour la conception - Dialectiques activités développement. (PP. 211-229). Toulouse : Octares.

Vérillon, P. (2005). Processus productifs et constructifs dans les activités physiques et sportives : la place de l'instrument. Impulsions, 4, 305-325.

Vigarello, G. (1988). Une histoire culturelle du sport. Techniques d'hier... et d'aujourd'hui. Paris : Revue EPS - Robert Laffont.

Vigarello, G. (1991). Pour une technologie culturelle dans le champ des pratiques sportives. In J. Ardoino \& J.M. Brohm (Eds). Anthropologie du sport. Perspectives critiques. Actes du colloque international francophone. Paris-Sorbonne, 19-20 avril 1991. (pp. 146-151). Andsha - Matrice - Quel Corps ? 